\title{
Model for Predicting Bank Loan Default using XGBoost
}

\author{
Omogbhemhe M.I. \\ Department of Computer Science \\ Ambrose Alli University
}

\author{
Momodu I.B.A. \\ Department of Computer Science \\ Ambrose Alli University
}

\begin{abstract}
Loan default prediction is one of the most important and critical problem faced by many banks and other financial institutions as it has a huge effect on their survival and profit. Many traditional methods exist for mining information about a loan application and have been greatly studied and applied in the past. These methods seem to be underperforming as there have been reported increases in the amount of bad loans and defaulters among many financial institutions. In this paper, gradient boosting algorithm called XGBoost was used for loan default prediction. The prediction is based on a loan data from a leading bank taking into consideration data sets from both the loan application and the demographic of the applicant. Similarly, important evaluation metrics such as Accuracy, Recall, precision, F1-Score and ROC area of the analysis were used. The paper provides an effective basis for loan credit approval in order to identify risky customers from a large number of loan applications using predictive modeling. The full utilization of this model will assist financial institutions in knowing a risking customer that may default in loan payment before lending.
\end{abstract}

\section{Keywords}

XGBoost, Bank Loan Default

\section{INTRODUCTION}

The credit lending business of the banking industry has seen rapid progress and extreme competitions from numerous credit startups. At the same time, the increase in credit application and consumption has also led to the increase in losses resulting from bad credits.

Credit loan refers to credits provided by banks or financial institutions in general to individuals/consumers which is payable at an agreed date with/without a specified interest.

Credit loans are usually provided for many purposes some of which are personal use, educational purpose, medical purpose, travelling and business purposes.

The increase in the application for loans plus the rapidly growing competition means financial institutions must build effective models that can capture the information in the available data, and create robust predictive models that can help minimize the chances of bad credit.

Through numerous modern predictive modeling, financial institutions can get insights into applicant's behavior, consumption patterns, default predictors and characteristics. Numerous studies have been conducted in order to identify the important factors that can affect the loan repayment, these studies are important as they help to maximize profit for banks. According to [1], there are seven types of variables that may influence consumer loan default; these are relationship between consumer and creditors, consumer's annual income, debt-income ratio, occupation, house ownership, work duration and whether or not consumer possesses a saving/checking account.

[2] also indicated that an applicant's individual characteristics such as age, attitude could also influence risk behavior of borrowers.

In a work by [3], the key factors that may influence loan default are borrower's age, location, resident/work duration, owner of phone, monthly income, loan duration, whether or not applicant works in a public sector, house ownership and loan numbers.

Another study by [4], on a large dataset within the period of 2001-2006 indicated that loan age was the most important factor when predicting loan default while market loan-tovalue was the most effective factor for mortgage loan applications.

In addition to identifying factors that may influence loan default, there is also a need to build robust and effective machine learning models that can help capture important patterns in credit data. The choice of model is of great importance as the chosen model plays a crucial role in determining accuracy, precision and efficiency of a prediction system. Numerous models have been used for loan default prediction and although there is no one optimal model, some models definitely do better than others.

[1] used a Neural Network to predict loan default on data from a lending club bank and inferred that a Neural Network performs better than most traditional models.

[5] did a comparative study of three models (Random Forest, Logistic Regression and Support Vector Machines) on bank credit data and found out that Random Forest does better at the task.

[4] applied four algorithms to a large dataset of about 20 million observations and compared the result with a logistic regression model-a widely used traditional model for classification task.

Yang et al used a Logistic Regression model on a credit company's data to identify the most influential corporate financial indicators.

[6] conducted a study on data from a credit company in Taiwan, and compared different models such as CART, Neural Networks, Linear regression etc. The study concluded that the CART had better accuracy than the other models.

In this paper, we use a gradient boosting algorithm XGBoost to study and analyze bank loan dataset and suggest some of the important factors/variables that may influence loan repayment. We also present evaluation statistics (Accuracy, Precision, Recall, Confusion Matrix, f1-Score ad ROC area) of our model.

\section{BACKGROUND}

In this section, we describe the machine learning algorithm we use to forecast credit loan default in a supervised machine learning setting. In Supervised learning setting, a predictor/learner/estimator is presented with input-output pairs $\left\{\left(x_{1}, y_{1}\right),\left(x_{1}, y_{1}\right), \ldots,\left(x_{n}, y_{n}\right)\right\}$ for some function $\mathrm{y}=\mathrm{f}(\mathrm{x})$. In this analysis, the supervised learning problem is posed as a classification problem since the target/output is composed of discrete binary variables (good/bad credit). There exist many machine learning algorithms such as Logistic Regression, Neural Networks, 
Vector Machines, Decision Trees etc. that can be used for this classification task but we use a technique of ensembling called Gradient Boosting (XGBoost) as these have been shown to outperform many traditional algorithms in structured data settings like we have here.

\subsection{Overview of Boosting}

Boosting is a popular ensemble technique that originated in answer to a question posed in this form. That is, whether a "weak learner" could be made better by using some form of modification. This is similar to the statistical question of whether it is possible to create a "good hypothesis" from a "poor hypothesis". This was discovered to be possible and the first boosting algorithm Adaptive Boosting (AdaBoost) was created by [7].

The concept of boosting is to correct the mistakes made by earlier learners and improving on those areas [8]. In this paper the new base learners that are more correlated to the negative gradient of our objective function was constructed.

Boosting can also be seen as a kind of stage wise "additive modeling in that it is an additive combination of a simple base estimator. Boosting is similar to bagging; the only difference is how they are trained.

Gradient Boosting is a type of boosting where the objective is treated as optimization problem and training is done using weight updates by gradient descent.

\section{Algorithm I Friedman's Gradient Boosting Algorithm}

\section{Inputs:}

- Input data $(\mathrm{x}, \mathrm{y}) \mathrm{N} \mathrm{i}=1$

- Number of iterations $\mathrm{M}$

- Choice of the loss-function (y, f)

- Choice of the base-learner model h(x, $\theta)$

\section{Algorithm:}

1: initialize $\mathrm{f}_{0}$ with a constant

2: for $t=1$ to $M$ do

3: $\quad$ compute the negative gradient $\mathrm{g}_{\mathrm{t}}(\mathrm{x})$

4: fit a new base-learner function $\mathrm{h}\left(\mathrm{x}, \theta_{\mathrm{t}}\right)$

5: find the best gradient descent step-size $\rho_{\mathrm{t}}$ :

$$
\rho_{\mathrm{t}}=\arg \min _{\rho} \sum_{i=1}^{N} \varphi[y i, f i-1(x i)+\rho h(x i, \theta t)]
$$

6: update the function estimate:

$$
f_{t} \leftarrow f_{t}-1+\rho_{t} h\left(x, \theta_{t}\right)
$$

\section{7: end for}

\subsection{XGBoost}

XGBoost-Extreme Gradient Boosting [9][10] is a scalable and highly efficient boosting system. It has been shown to achieve state-of-the-art results on many machine learning tasks. In XGBoost algorithm, unlike the traditional gradient boosting, the process of adding weak learners does not happen sequentially; it approaches this phase in parallel using a multithreaded pattern, thereby resulting in proper utilization of hardware resources leading to greater speed and efficiency. Some important features that make XGBoost more efficient than traditional boosting algorithms are:

1. Sparse aware implementation.

2. Weighted quartile sketch for approximate tree learning.
3. Cache-aware access.

4. Blocks for out-of-core computation.

\subsection{Data}

This paper used the loan credit data set provided by Data Science Nigeria and hosted on the Zindi platform. There are three set of data:

1. Demographic data: This contains description and details about loan applicants. Attributes such as birthdates, addresses and account types can be found here. The data is made up of 4346 samples and 9 attribute features.

2. Performance data: This contains further details of each applicant and a feature (good_bad_flag) which describes if an applicant is a good or bad debtor. The data contains 4368 samples and 10 attributes.

3. Previous loan data: This contains all previous loan applications. The data contains 18183 samples and 12 attributes.

Table 1 Description data sets

\begin{tabular}{|l|l|l|}
\hline \multicolumn{1}{|c|}{ Data } & \multicolumn{1}{|c|}{$\begin{array}{c}\text { No of } \\
\text { Attributes }\end{array}$} & \multicolumn{1}{c|}{ Samples } \\
\hline Demographic & 9 & 4346 \\
\hline Loan performance & 10 & 4368 \\
\hline Previous Loan & 12 & 18183 \\
\hline
\end{tabular}

\section{Preparing the data set}

After collecting the data, we performed data processing and wrangling. The framework for preprocessing the data sets is shown below. 


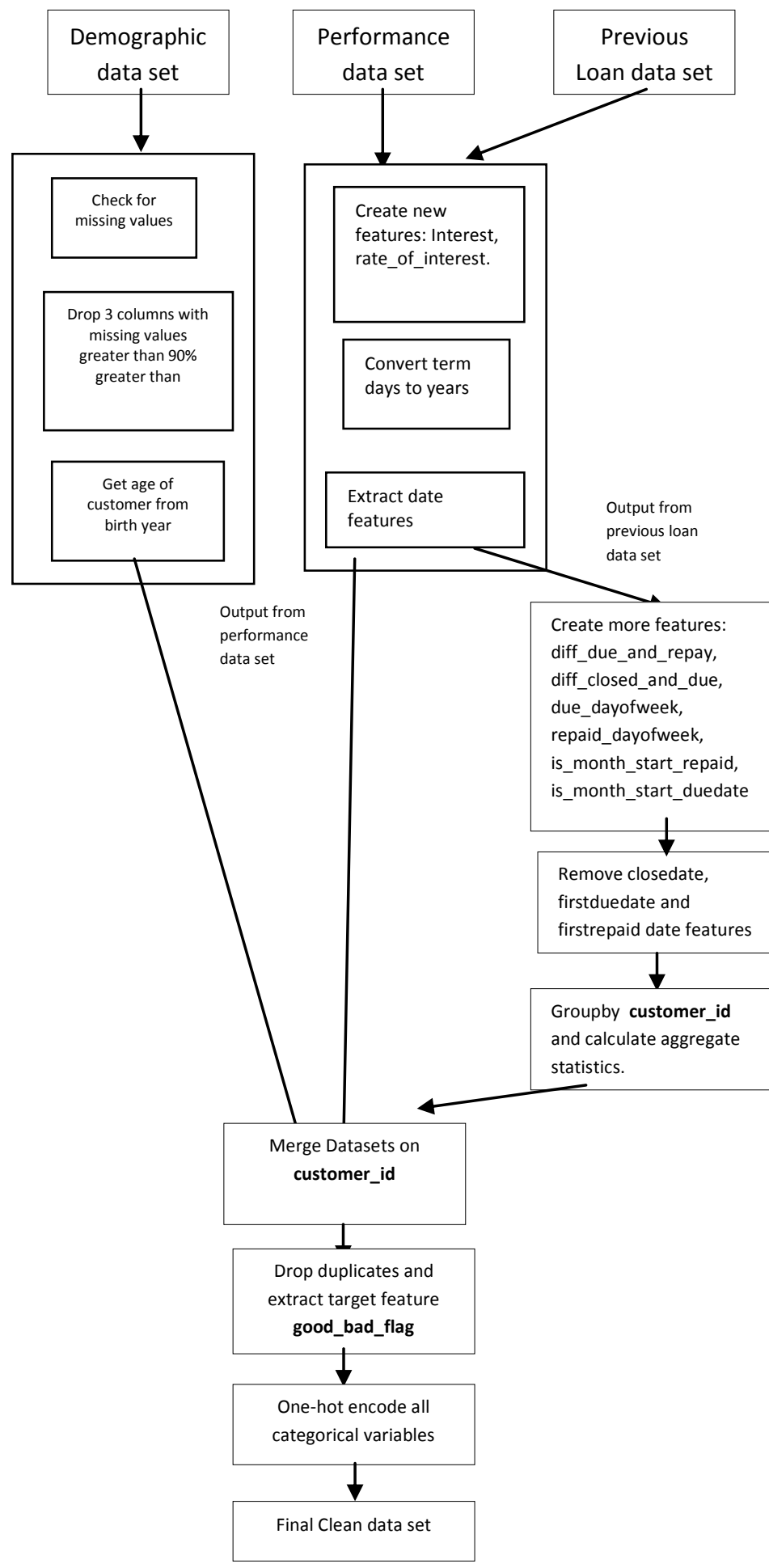

Figure1 Flowchart for data preprocessing

\section{Findings and Discussions}

Based on the pre-processed data sets, the XGBoost Classifier algorithm was used and implemented in python programming language. The classifier was trained on the clean data set using the feature good_bad_flag as target. The paper used a 5 fold cross validation to avoid recording a bias performance. Optimal parameters were obtained using a Grid Search.

For evaluation performance, we use five metrics; Accuracy, F1-Score, Recall, Precision and ROC area.
Table 2 Optimal parameters for our algorithm after Gridsearch

\begin{tabular}{|l|l|}
\hline Parameter & Value \\
\hline n_estimators & 1000 \\
\hline class_weight & binary \\
\hline learning_rate & 0.01 \\
\hline sub_sample & 0.8 \\
\hline reg_alpha & 1 \\
\hline reg_lambda & 1 \\
\hline max_depth & 6 \\
\hline
\end{tabular}

\section{Confusion Matrix}

The confusion matrix is an important 2 dimensional matrix that contains information about the actual classes and the predicted classes of a classifier. In the loan application data used in this paper, the number 0 represents the loan default category, and 1 represent the normal category. Table 3 shows the calculated confusion matrix of XGBoost the data.

Table 3 Confusion Matrix of XGBoost

\begin{tabular}{|c|c|c|c|}
\hline & \multicolumn{3}{|c|}{ Predicted Class } \\
\hline \multirow{3}{*}{$\begin{array}{l}\text { Actual } \\
\text { Class }\end{array}$} & & $\begin{array}{l}\text { Class }= \\
\text { good }\end{array}$ & Class $=$ bad \\
\hline & $\begin{array}{l}\text { Class }= \\
\text { good }\end{array}$ & 175 & 15 \\
\hline & Class $=$ bad & 14 & 670 \\
\hline
\end{tabular}

\section{Accuracy}

Accuracy measures the proportion of correctly classified predictions; it is defined by the formula:

Accuracy $=\frac{T P+T N}{T P+T N+F P+F N}$

Where TP is the number of true positives, $\mathrm{TN}$ is the number of true negatives, FP is the number of false positives and FN is the number of false negatives.

Recall

Recall measures the ability of the classifier to predict correctly instances of a certain class; it is also called the TPR (true positive rate):

Recall $=\frac{T P}{T P+F N}$

\section{Precision}

Precision measures the proportion of predictions made by the classifier as positive that are actually positive:

Precision $=\frac{T P}{T P+F P}$

\section{F1-score}

F1-score is the harmonic average of precision and recall:

F1-score $=\frac{2 * \text { Precision } * \text { Recall }}{\text { Precision }+ \text { Recall }}$

Table 4 Performance metric for XGBoost algorithm on the loan dataset

\begin{tabular}{|l|l|}
\hline Metric & Score (\%) \\
\hline Accuracy & 79 \\
\hline Precision & 97 \\
\hline Recall & 79 \\
\hline F1_score & 87 \\
\hline
\end{tabular}




\section{Receiver Operating Characteristic Curve (ROC)}

ROC is a visualization technique for showing a classifier's performance. It represents the sensitivity and specificity of the classifier. The ROC curve is a two-dimensional curve with the FPR (false positive rate) as the $\mathrm{X}$-axis and the TPR (true positive rate) as the $\mathrm{Y}$-axis. The ranges of the ROC curve runs from $(0,0)$ to $(1,1)$. To compare models, we calculate the area under the ROC curve (AUC). The larger the AUC, the better the model.

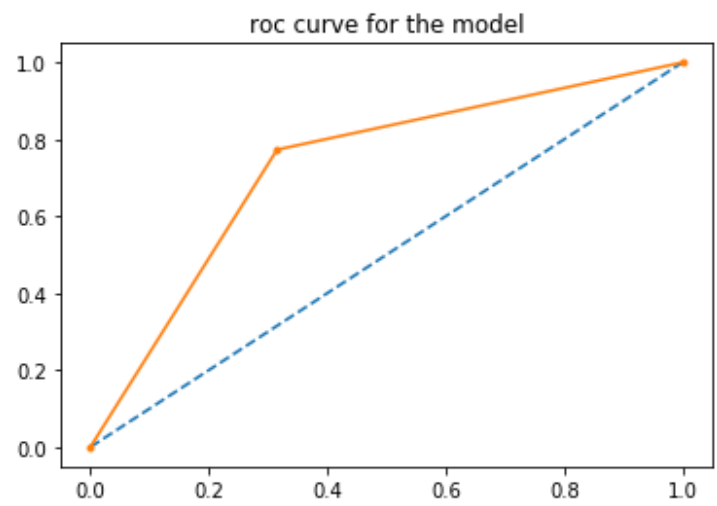

Figure 2 ROC area curve of XGBoost on the loan data set.

\subsection{Feature Importance}

One of the important results of this study is to determine the important features that help the classifier to correctly predict loan default. This helps in business intelligence and decision making. Figure 3 shows the top 10 most important features. The rate of loan interest (rateofinterest) is the most important feature from our analysis.

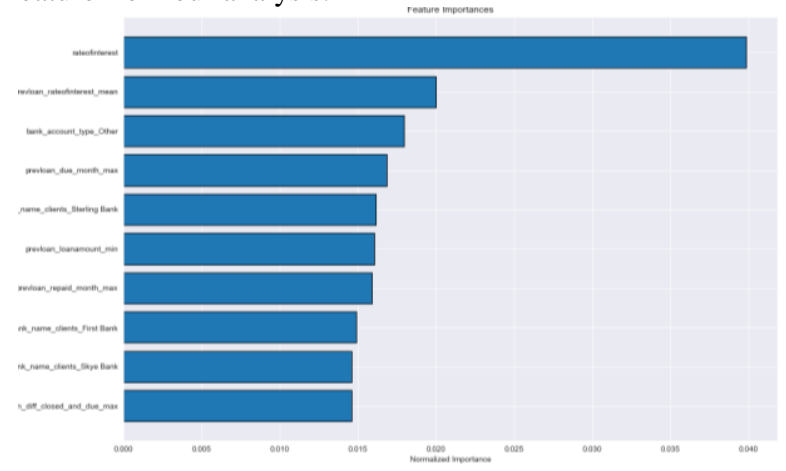

\section{CONCLUSION}

In this paper, XGBoost on loan default prediction was used, the task of predicting if a loan applicant will default in loan payment. The analysis was done using a boosting algorithm called XGBoost implemented in python programming language and five performance metrics was calculated: Accuracy, Recall, Precision, F1-score and ROC area. The most important feature was identified to be rate of interest. This paper provides an effective basis for loan credit approval in order to identify risky customers from a large number of loan applicants using predictive modeling.

\section{REFERENCES}

[1] Manjeet K, Vishesh G, Tarun J, Sahil S and Lalit M. G. (2018). Neural Network Approach To Loan Default Prediction, International Research Journal of Engineering and Technology (IRJET), p-ISSN: 2395-0072.

[2] Chiang, R. C., Chow, Y. F., \& Liu, M. (2002). Residential mortgage lending and borrower risk: The relationship between mortgage spreads and individual characteristics. Journal of Real Estate Finance and Economics, 25(1), 5-32.

[3] Steenackers, A., \& Goovaerts, M. J. (1989). A credit scoring model for personal loans. Insurance: Mathematics and Economics, 8(1), 31-34.

[4] Li Y. (2018). Research on bank credit default prediction based on data mining algorithm. The International Journal of Social Science and Humanities Invention 5(06): 4820-4820, ISSN: 2349-2031

[5] Ali B. (2006). Predicting Mortgage Loan Default with Machine Learning Methods.

[6] Lee, T. S., Chiu, C. C., Chou, Y. C., \& Lu, C. J. (2006). Mining the customer credit using classification and regression tree and multivariate adaptive regression splines. Computational Statistics and Data Analysis, 50, 111.

[7] Freund, Y., \& Schapire, R. (1996). Experiments with a new boosting algorithm. In Proceedings of the Thirteenth International Conference on Machine Learning, pp. 148156 Bari, Italy.

[8] Zhou Z.H and Li. M(2012) Ensemble Methods Foundations and Algorithms, -13: 978-1-4398-3005 -5.

[9] Jerome H. F (1999). Greedy Function Approximation: A Gradient Boosting Machine, IMS 1999 Reitz Lecture.

[10] Tianqi C and Carlos G. (2016). XGBoost: A scalable tree boosting system, arXiv:1603.02754[cs.LG] 